\title{
Vitamin D Supplementation is Associated with Disease Activity in Systemic Lupus Erythematosus Patients ${ }^{+}$
}

\author{
María Correa-Rodríguez ${ }^{1,2, *}$, Gabriela Pocovi-Gerardino ${ }^{1,2}$, Irene Medina-Martínez ${ }^{1}$, Sara Del \\ Olmo-Romero ${ }^{1}$, Norberto Ortego-Centeno ${ }^{2,3}$ and Blanca Rueda-Medina ${ }^{1,2}$ \\ 1 Department of Nursing, Health Sciences Faculty, University of Granada (UGR), Avenida de la Ilustración \\ s/n, 18100 Armilla (Granada), Spain; gpocovi@correo.ugr.es (G.P.-G.); irenemedina@correo.ugr.es (I.M.-M.); \\ sdelolmo@correo.ugr.es (S.D.O.-R.); blarume@ugr.es (B.R.-M.) \\ 2 Instituto de Investigación Biosanitaria, IBS. Avda. de Madrid, 15. Pabellón de consultas externas 2, 2 $2^{\text {a }}$ \\ planta, 18012 Granada, Spain; nortego@gmail.com \\ 3 Unidad de Enfermedades Autoinmunes Sistémicas, Servicio de Medicina Interna, Hospital Universitario \\ San Cecilio, Av. de la Investigación, s/n, 18016 Granada, Spain \\ * Correspondence: macoro@ugr.es; Tel.: +34-659-242-506 \\ + Presented at the 1st International Electronic Conference on Nutrients, 2-15 November 2020; Available \\ online: https://iecn2020.sciforum.net/.
}

Published: 30 October 2020

\begin{abstract}
Systemic lupus erythematosus (SLE) is a chronic disease characterized by inflammatory response and abnormal autoimmune disease. Vitamin D is essential in phosphorus-calcium metabolism and it has immunosuppressive properties, being considered as a therapeutic option. There is controversy about the role of this vitamin in the pathogenesis of SLE. Thus, the aim of this study was to investigate the influence of the dietary intake of vitamin D and supplementation of vitamin D in a cohort of patients with SLE. A cross-sectional study including a total 285 patients with SLE was conducted (248 females y 26 males; mean age $46.99 \pm 12.89$ years). The SLE Disease Activity Index (SLEDAI-2K) and the SDI Damage Index were used to asses disease activity and disease-related damage, respectively. Levels of C-reactive protein (CRP; $\mathrm{mg} / \mathrm{dL}$ ), homocysteine (Hcy;mol/L), anti-double stranded DNA antibodies (anti-dsDNA) (IU/mL), complement C3 $(\mathrm{mg} / \mathrm{dL})$, and complement $\mathrm{C} 4(\mathrm{mg} / \mathrm{dL})$, among other biochemical markers, were measured. The dietary intake of vitamin $\mathrm{D}$ and the intake of vitamin D supplement were obtained via a 24-h patient diary. $57.1 \%$ of the patients were taking vitamin $\mathrm{D}$ supplements and the average of dietary vitamin $\mathrm{D}$ was $2.08 \pm 2.94 \mu \mathrm{g} /$ day. Note that $98.2 \%$ of patients did not reach the recommended dietary intakes for vitamin D intake. Multivariate regression analysis revealed that clinical and laboratory variables are not significantly affected by vitamin $\mathrm{D}$ intake levels after adjusting for age, gender, energy intake, and medical treatment (immunosuppressants, corticosteroids and antimalarials). Patients with SLE who took vitamin D supplements had significantly higher serum levels complement $\mathrm{C} 3$ compared to patients who did not take them after adjusting for covariates (110.28 \pm 30.93 vs. $107.38 \pm 24.18 ; \mathrm{p}=0.018$ ). Our findings suggest a potential impact of supplementation of vitamin D on the activity of SLE. Future longitudinal research on SLE patients, including intervention trials, are required to validate these preliminary data.
\end{abstract}

Keywords: Autoimmune; Systemic Lupus Erythematosus; Vitamin D intake; Vitamin D supplementation

\section{Introduction}


Systemic lupus erythematosus (SLE) is a systemic autoimmune disease characterized by the presence of autoantibodies directed against nuclear antigens. It is a multisystem disease with heterogenous clinical manifestations including rash, arthritis, fatigue, nephritis, neurological problems, anaemia and thrombocytopaenia [1]. The aetiology of SLE is unknown although it has been proposed that multiple genetic, immunological, endocrine and environmental factors might play a main role in development and activity [2].

Vitamin D is essential in phosphorus-calcium metabolism and it has immunosuppressive properties, being considered as a therapeutic option in autoimmune diseases [3]. There is controversy about the role of this vitamin D in the pathogenesis of SLE although it has been established as an essential element that may be relevant in the progression [4]. Recent research have reported that vitamin intake might be linked to SLE since vitamin D deficiency is high prevalence in SLE patients $[4,5]$. Also, vitamin D intake was found to be associated with decreased risk of other autoimmune diseases such as multiple sclerosis [6].

Considering the inconsistent findings and limited evidence regarding potential association between vitamin D intake and SLE activity and taking into account that SLE has a significant impact of the mortality and morbidity of patients, we aimed to investigate the relationship between the dietary intake of vitamin D and supplementation of vitamin D and SLE disease in a cohort of patients with SLE.

\section{Methods}

\subsection{Study Population}

A cross-sectional study was conducted among a population of SLE patients recruited in the Andalusian region of Spain. All patients met the SLE revised criteria of the American College of Rheumatology (ACR) or Systemic Lupus Erythematosus International Collaborating Clinics Group (SLICC) criteria [7,8]. The exclusion criteria were: pregnancy, cerebrovascular disease, ischemic heart disease, active infections, major trauma or surgery in the previous six months, serum creatinine $\geq 1.5$ $\mathrm{mg} / \mathrm{dl}$ and the presence of other autoimmune and/or chronic diseases not related with the main disease (i.e type 1 diabetes, multiple sclerosis rheumatoid arthritis, cancer ). A total of 258 SLE meet the inclusion criteria and were included in the study after giving written informed consent (mean age of $46.99 \pm 12.89$; $90.5 \%$ female). Local ethics committees approved the study protocol ("Comité Coordinador de Ética de la Investigación en Andalucía" (30-11-2016)) that was conducted in agreement with the Declaration of Helsinki.

\subsection{SLEDAI and SDI assessment}

The activity of the disease was assessed with the Systemic Lupus Erythematosus Disease Activity Index 2000 (SLEDAI-2K) [9]. The SLICC/ACR (SDI) damage index was used to measure disease-related organ damage [10].

\subsection{Assay of anti-dsDNA, complement levels, hs-CRP and Hcy determinations}

A BioPlex 2200 System (Bio-Rad, Hercules, CA, USA) which automatically detects antibodies to several antigens in one tube, was used to measure Anti-dsDNA titres. Serum samples were obtained to determine quantitatively human complement components $\mathrm{C} 3$ and $\mathrm{C} 4$ by immunoturbidimetric assay (Beckman Coulter AU System CRP Latex reagent) using a Beckman Coulter analyser (AU5800 Analyzer, Beckman Coulter, CA, USA). A highly sensitive technique based on immunoturbidimetric assays was used to measure hs-CRP levels (Beckman Coulter AU System CRP Latex reagent) in a Beckman Coulter analyser (AU5800 Analyzer, Beckman Coulter, CA, USA). An enzymatic colorimetric assay was used to determine Hcy serum levels in a Beckman Coulter analyser (AU680, Beckman Coulter, CA, USA) with the Axis-Shield Liquid Stable (LS) 2-Part Homocysteine Reagent (Axis-Shield Diagnostics Ltd., Dundee, UK).

\subsection{Dietary intake of vitamin $D$ and vitamin $D$ supplementation}


To estimate dietary intake of vitamin D and vitamin D supplementation a 24-h diet recall was used. Pictorial food models and standard household measures were used for a better exactness of the food quantities registered [11]. To convert the reported food and beverage intake into nutrient intake the software "El Alimentador" (Fundación Alimentación Saludable. Madrid, Spain) [12] was used.

\subsection{Statistical Analysis}

All statistical analyses were conducted using the SPSS ${ }^{\circledR}$ Statistics version 21.0 (SPSS, Chicago, IL, USA) software. Categorical variables were expressed as frequencies and percentages and continuous variables as mean \pm standard deviation To verify data distribution normality the KolmogorovSmirnov test was applied. Multivariate regression analysis were used to analyze the association between clinical disease variables and the intake of vitamin $\mathrm{D}$ after adjusting for the following covariates: age, sex, energy intake and medical treatments (immunosuppressants, antimalarials and/or corticosteroids. P values of $<0.05$ were taken as statistically significant.

\section{Results}

The main characteristics of the study population are shown in Table $1.57 .1 \%$ of the patients were taking vitamin D supplements and the average of dietary vitamin D was $2.08 \pm 2.94 \mu \mathrm{g} /$ day. Note that $98.2 \%$ of patients did not reach the recommended dietary intakes for vitamin D intakeThe introduction should briefly place the study in a broad context and define the purpose of the work and its significance.

Table 1. Descriptive of the main characteristics the study population.

\begin{tabular}{lc}
\hline \multicolumn{1}{c}{ Characteristics } & $\begin{array}{c}\text { Total } \\
(\mathbf{n}=\mathbf{1 9 3})\end{array}$ \\
\hline Female & $248(90.5)$ \\
Age (years) & $46.99 \pm 12.89$ \\
Energy (kcal) & $1775.03 \pm 507.69$ \\
Vitamin D intake $(\mu \mathrm{g})$ & $2.08 \pm 2.94$ \\
Clinical data & \\
Time since diagnosis (years) & $9.11 \pm 6.64$ \\
Number of complications & $3.38 \pm 1.34$ \\
SLEDAIa score & $2.65 \pm 2.68$ \\
SDI score & $0.97 \pm 1.23$ \\
Laboratory markers & \\
hsCRP (mg/dL) & $3.17 \pm 4.81$ \\
Hcy ( $\mu$ mol/L) & $12.48 \pm 7.45$ \\
Anti-dsDNA (IU/mL) & $18.37 \pm 37.09$ \\
Complement C3 level (mg/dL) & $109.17 \pm 28.25$ \\
Complement C4 level (mg/dL) & $22.81 \pm 13.56$ \\
Medication used & \\
Vitamin D supplementation & $156(57.1 \%)$ \\
Antimalarial use & $217(79.5)$ \\
Immunosuppressor use & $102(37.4)$ \\
Corticoid use & $108(39.6)$ \\
\hline
\end{tabular}

Data are expressed as mean and range or frequency and percentage. SLEDAI= systemic lupus erythematosus disease activity index; $\mathrm{SDI}=$ damage index for systemic lupus erythematosus; hsCRP= high-sensitivity C-reactive protein; Hcy= homocysteine; Anti-dsDNA= Anti-double stranded DNA antibodies.

Multivariate regression analysis revealed that clinical and laboratory variables are not significantly affected by vitamin D intake levels after adjusting for age, gender, energy intake, and medical treatment (immunosuppressants, corticosteroids and antimalarials) (Table 2). In contrast, 
patients with SLE who took vitamin D supplements had significantly higher serum levels complement $\mathrm{C} 3$ compared to patients who did not take them after adjusting for covariates $(110.28 \pm$ 30.93 vs. $107.38 \pm 24.18 ; \mathrm{p}=0.018$ ) (Table 2). Unexpectedly, also patients with SLE who took vitamin D supplements had significantly higher homocysteine levels compared to patients who did not take them $(13.66 \pm 9.50$ vs. $11.05 \pm 3.18 ; \mathrm{p}=0.016)$.

Table 2. Beta estimates and confidence intervals for the association between vitamin D intake, vitamin D supplementation and clinical disease activity parameters in SLE patients.

\begin{tabular}{cccccc}
\hline & \multicolumn{2}{c}{ Vitamin D Intake $(\mu \mathrm{g})$} & \multicolumn{2}{c}{ Vitamin D Supplementation } \\
\hline Clinical Parameters & $\boldsymbol{\beta}(\mathbf{9 5} \% \mathbf{C I})$ & $\boldsymbol{P}$ Value & Yes (n=156) & No (n= 117) & $\boldsymbol{P}$ Value \\
\hline Number of complications & $0.004(-0.049,0.056)$ & 0.895 & $3.53 \pm 1.44$ & $3.17 \pm 1.18$ & 0.922 \\
SLEDAI score & $0.015(-0.092,0.121)$ & 0.784 & $2.68 \pm 2.85$ & $2.57 \pm 2.40$ & 0.229 \\
SDI score & $0.012(-0.036,0.060)$ & 0.623 & $1.19 \pm 1.35$ & $0.66 \pm 0.96$ & 0.123 \\
hsCRP (mg/dL) & $-0.108(-0.301,0.086)$ & 0.274 & $3.76 \pm 5.54$ & $2.29 \pm 3.37$ & 0.238 \\
Hcy ( $\mu$ mol/L) & $0.339(-0.052,0.731)$ & 0.089 & $13.66 \pm 9.50$ & $11.05 \pm 3.18$ & $\mathbf{0 . 0 1 6}$ \\
Anti-dsDNA (IU/mL) & $0.493(-1.013,1.999)$ & 0.520 & $21.33 \pm 43.41$ & $14.54 \pm 26.12$ & 0.938 \\
$\begin{array}{c}\text { Complement C3 level } \\
\text { (mg/dL) }\end{array}$ & $-0.759(-1.869,0.351)$ & 0.180 & $110.28 \pm 30.93$ & $107.38 \pm 24.18$ & $\mathbf{0 . 0 1 8}$ \\
$\begin{array}{c}\text { Complement C4 level } \\
\text { (mg/dL) }\end{array}$ & $-0.433(-1.128,0.261)$ & 0.220 & $22.53 \pm 12.94$ & $23.16 \pm 21.41$ & 0.894 \\
\hline
\end{tabular}

SLE= systemic lupus eritematosus; SLEDAI= systemic lupus erythematosus disease activity index; $\mathrm{SDI}=$ damage index for systemic lupus erythematosus; hsCRP= high-sensitivity C-reactive protein; Hcy= homocysteine; Anti-dsDNA= Anti-double stranded DNA antibodies. Data were adjusted by age, sex, energy intake and medical treatment (immunosuppressor, corticoid and antimalarial use).

\section{Discussion}

In the present study we evidenced that the supplementation of vitamin D might have a potential impact on the activity of SLE since we found that patients taking vitamin D supplements had significantly higher levels of complement 3 compared to patients not supplemented. Unexpectedly, we also found that patients with SLE who took vitamin D supplements had significantly higher homocysteine levels. Research in SLE are very limited and usually based on a small sample size and have led to inconsistent findings. In contrast to our results, Mellor-Pita el at reported no association between vitamin D supplement intake and SLE-related factors including homocysteine in forty-seven females with SLE [13]. These discrepancies could be due to differences in the sample size or to heterogeneity in the clinical characteristics of the recruited patients. Also, it should be mentioned that our study included a well-characterised cohort of population with SLE, including patients in an earlystage of the disease and excluding any with lupus severe complications or affected by other autoimmune diseases. However, in previous studies this data has not been specified [13]. Thus, taking into account that the limited prior studies carried out to investigate the effect of vitamin $\mathrm{D}$ supplementation on SLE have produced inconsistent findings and in order to validate our preliminary results, future studies in homogeneous cohort of SLE patients are required.

On the other hand, in line with previous studies, we observed that dietary intake of vitamin D are not associated with clinical and laboratory variables [5]. This finding support the hypothesis that the dietary intake of vitamin D might have no effect on SLE disease activity. Among the same line, Costenbader et al. conclude that vitamin D intake was not associated with risk of SLE in a large prospective cohort of women [5].

This study has some limitations that should be mentioned. Firstly, it was cross-sectional study and, as such, was subject to the limitations inherent to this type of design. Secondly, there were also certain limitations derived from the use of the 24 -h diet recall technique because it is prone to under- 
reporting and relies on participant memory [14]. In contrast, it should be noted that we included the use of medications (antimalarials, immunosuppressors, and corticosteroids) as well as age, sex, and energy intake, as cofounders because these factors could have influenced the results.

\section{Conclusions}

In conclusion, patients taking vitamin $\mathrm{D}$ supplements had significantly higher levels of complement 3 compared to patients not supplemented, supporting the potential effect of supplementation of vitamin D on the activity of SLE. However, the dietary intake of vitamin D are not associated with clinical and laboratory variables in women with SLE.

Author Contributions: Data curation, M.C.-R., G.P.-G., S.D.O.-R. and I.M.-M.; Formal analysis, M.C.-R.; Funding acquisition, N.O.-C. and B.R.-M.; Investigation, G.P.-G., and S.D.O.-R.; Methodology, G.P.-G., and I.M.M.; Project administration, B.R.-M.; Resources, B.R.-M.; Software, I.M.-M.; Supervision and N.O.-C.; Validation, M.C.-R.; Writing - original draft, M.C.-R.; Writing - review \& editing, N.O.-C. and B.R.-M. All authors have read and agreed to the published version of the manuscript.

Fundings: This research was supported by the grant PI0523-2016 from “Consejería de igualdad, salud y políticas sociales" (Junta de Andalucía) and is part of the research group LyDIMED “Lupus y Dieta Mediterránea".

Conflicts of Interest: The authors declare no conflict of interest.

\section{References}

1. Fava, A.; Petri, M. Systemic lupus erythematosus: Diagnosis and clinical management. J. Autoimmun 2019, 96, 1-13.

2. Rekvig, O.P. Systemic lupus erythematosus: Definitions, contexts, conflicts, enigmas. Front. Immunol. 2018, 9, 1, doi:10.3389/fimmu.2018.00387.

3. Yang, C.Y.; Leung, P.S.C.; Adamopoulos, I.E.; Gershwin, M.E. The implication of vitamin D and autoimmunity: A comprehensive review. Clin. Rev. Allergy Immunol. 2013, 45, 217-226.

4. Hassanalilou, T.; Khalili, L.; Ghavamzadeh, S.; Shokri, A.; Payahoo, L.; Bishak, Y.K. Role of vitamin D deficiency in systemic lupus erythematosus incidence and aggravation. Autoimmun. Highlights 2018, 9, 110, doi:10.1007/s13317-017-0101-x.

5. Costenbader, K.H.; Feskanich, D.; Holmes, M.; Karlson, E.W.; Benito-Garcia, E. Vitamin D intake and risks of systemic lupus erythematosus and rheumatoid arthritis in women. Ann. Rheum Dis. 2008, 67, 530-535, doi:10.1136/ard.2007.072736.

6. Munger, K.L.; Zhang, S.M.; O’Reilly, E.; Hernán, M.A.; Olek, M.J.; Willett, W.C.; Ascherio, A. Vitamin D intake and incidence of multiple sclerosis. Neurology 2004, 62, 60-65, doi:10.1212/01.WNL.0000101723.79681.38.

7. Hochberg, M.C. Updating the American College of Rheumatology Revised criteria for the classification of Systemic Lupus Erythematosus. Arthritis Rheum 1997, 40, 1997, doi:10.1002/15290131(200103)44:3<735::AID-ANR125>3.0.CO;2-F.

8. Petri, M.; Orbai, A.M.; Alarcõn, G.S.; Gordon, C.; Merrill, J.T.; Fortin, P.R.; Bruce, I.N.; Isenberg, D.; Wallace, D.J.; Nived, O.; et al. Derivation and validation of the systemic lupus international collaborating clinics classification criteria for systemic lupus erythematosus. Arthritis Rheum 2012, 64, 2677-2686, doi:10.1002/art.34473.

9. Gladman, D.D.; Ibañez, D.; Urowitz, M.B. Systemic lupus erythematosus disease activity index 2000. J. Rheumatol. 2002, 29, 288-291, doi:10.3899/jrheum.110550.

10. Gladman, D.; Ginzler, E.; Goldsmith, C.; Fortin, P.; Liang, M.; Urowitz, M.; Bacon, P.; Bombardieri, S.; Hanly, J.; Hay, E.; et al. The development and initial validation of the Systemic Lupus International Collaborating Clinics/American College of Rheumatology damage index for systemic lupus erythematosus. Arthritis Rheum 1996, 39, 363-369.

11. Ruiz MD, M.-L. R. Guia Para Estudios Dieteticos. Album Fotografico de Alimentos; 1st ed.; Universidad de Granada: Granada, España, 2010.

12. Martínez Álvarez, J.R.; Villarino Marín, A.; García Alcón, R.; Fernando Martín, F. Aplicación Online de Cálculo de Dietas Personalizadas; El Alimentador: 2019. 
13. Mellor-Pita, S.; Tutor-Ureta, P.; Rosado, S.; Alkadi, K.; Granado, F.; Jimenez-Ortiz, C.; Castejon, R. Calcium and vitamin $\mathrm{D}$ supplement intake may increase arterial stiffness in systemic lupus erythematosus patients. Clin. Rheumatol. 2019, 38, 1177-1186, doi:10.1007/s10067-018-04416-x.

14. Shim, J.-S.; Oh, K.; Kim, H.C. Dietary assessment methods in epidemiologic studies. Epidemiol. Health 2014, 36, e2014009, doi:10.4178/epih/e2014009.

Publisher's Note: MDPI stays neutral with regard to jurisdictional claims in published maps and institutional affiliations.

(C) 2020 by the authors. Submitted for possible open access publication under the terms and conditions of the Creative Commons Attribution (CC BY) license (http://creativecommons.org/licenses/by/4.0/). 